\title{
Optimizing the wireless power transfer over MIMO Channels
}

\author{
Karsten Wiedmann and Tobias Weber \\ Institute of Communications Engineering, University of Rostock, Richard-Wagner-Str. 31, 18119 Rostock, Germany \\ Correspondence to: Karsten Wiedmann (karsten.wiedmann@uni-rostock.de) \\ and Tobias Weber (tobias.weber@uni-rostock.de)
}

Received: 6 December 2016 - Accepted: 27 March 2017 - Published: 21 September 2017

\begin{abstract}
In this paper, the optimization of the power transfer over wireless channels having multiple-inputs and multiple-outputs (MIMO) is studied. Therefore, the transmitter, the receiver and the MIMO channel are modeled as multiports. The power transfer efficiency is described by a Rayleigh quotient, which is a function of the channel's scattering parameters and the incident waves from both transmitter and receiver side. This way, the power transfer efficiency can be maximized analytically by solving a generalized eigenvalue problem, which is deduced from the Rayleigh quotient.

As a result, the maximum power transfer efficiency achievable over a given MIMO channel is obtained. This maximum can be used as a performance bound in order to benchmark wireless power transfer systems. Furthermore, the optimal operating point which achieves this maximum will be obtained. The optimal operating point will be described by the complex amplitudes of the optimal incident and reflected waves of the MIMO channel. This supports the design of the optimal transmitter and receiver multiports.

The proposed method applies for arbitrary MIMO channels, taking transmitter-side and/or receiver-side crosscouplings in both near- and farfield scenarios into consideration. Special cases are briefly discussed in this paper in order to illustrate the method.
\end{abstract}

\section{Introduction}

The efficiency of wireless power transfer (WPT) systems is a crucial figure of merit. In order to optimize and/or assess WPT systems, the maximum power transfer efficiency (PTE), which can be achieved over a given channel, has to be investigated. This gives rise to an optimization problem since the maximum PTE as well as the optimum channel in- put and output, i.e., the optimal operating point, are not apriori known in general.

In the following, the WPT over channels having multipleinputs and multiple-outputs (MIMO) is considered. In order to formulate the optimization problem, the MIMO channel can be modeled as a multiport (see e.g., Ivrlac and Nossek, 2010; Wallace and Jensen, 2014). This allows to define the PTE as the ratio of the dissipated power in the load at the receiver over the total transmitted power at the transmitter side (Hui et al., 2014). This approach is adopted by Zhang and Ho (2013) to study the WPT over unidirectional MIMO channels. As a result, the PTE is maximized by transmitter side "energy" beamforming if full channel state information is available. A discussion on the PTE maximization in the case of imperfect channel state information can also be found in the literature (e.g., Xiang and Tao, 2012; Xu and Zhang, 2016). However, the aforementioned approaches require a perfectly decoupled transmit and receive antenna array, respectively. Further, the unidirectional channel approximation presumes that the waves at the transmitter side are not influenced by the waves at the receiver side, which holds approximately true only for farfield WPT (Wallace and Jensen, 2014). Contrarily, Lee and Nam (2010) and Zargham and Gulak (2012) discuss the maximum PTE of nearfield coupled single-input and single-output (SISO) channels. Similar investigations are proposed by Yoon and Ling (2011) and Lang et al. (2014) for MISO channels or by Kim et al. (2010) for SIMO channels. But an extension to general MIMO channels cannot be found and often numerical tools are applied to analyze the PTE of corresponding channels (e.g., Nguyen et al., 2014). Furthermore, the aforementioned approaches describe the channel by its impedance matrix, which is rather unfavorable from the measurement point of view. Alternatively, Yuan et al. (2010, 2013) and Wu et al. (2013) formulate the PTE of a $2 \times 2$ MIMO channel as a function of its scattering parame- 
ters. Unfortunately, an analytical solution of the optimization problem was not presented.

As can be seen, for the best knowledge of the authors, the WPT optimization over general MIMO channels is not sufficiently investigated in the literature so that basic insights regarding the PTE maximization over MIMO channels are missing, e.g., the influence of transmitter-side and/or receiver-side cross-couplings.

The contribution of this paper is in the investigation of the optimal operating point, which maximizes the PTE over a given MIMO channel. The optimal operating point will be described by the complex amplitudes of the optimal incident and reflected waves of the MIMO channel. Considering $N$ transmit antennas and $M$ receive antennas, the transmitter is modeled as an active $N$-port and the receiver as a passive $M$ port so that the MIMO channel can be modeled as a passive $(N+M)$-port. It is assumed that the scattering parameters are fully known, e.g., by measurement or simulation. No further restrictions on the WPT systems under investigation are required.

In order to find the optimum operating point, the PTE definition stated in the aforementioned literature is adopted. As Yuan et al. (2013) for the $2 \times 2$ system, the MIMO channel is described by its scattering parameters yielding a Rayleigh quotient. Different to the approach of Yuan et al. (2013), the derived Rayleigh quotient will be reformulated as a function of the scattering parameters of the MIMO channel and the incident waves from both transmitter and receiver side. The Rayleigh quotient will be solved by determining the eigenvalues of a generalized eigenvalue problem, which deduces from the derived Rayleigh quotient. Fortunately, the solution yields both the maximum PTE and the corresponding optimal incident and reflected waves that achieve the maximum.

As will be discussed, the proposed optimization framework applies for arbitrary MIMO channels taking transmitter-side and/or receiver-side cross-couplings in both near- and farfield scenarios into consideration. However, if the scattering matrix features certain properties, e.g., due to matched ports or due to the absence of antenna array crosscouplings, the mathematical effort reduces significantly and the outcomes of the proposed method coincide with the specific solutions in the literature.

The optimization framework can be used to gain insights in the WPT over arbitrary MIMO channels so that the design of the optimal transmitter and receiver multiports is supported. Furthermore, if only suboptimal transmitter and/or receiver multiports can be applied, the herein derived PTE can be regarded as a performance bound, which can be used in order to assess and/or enhance WPT systems.

Structure Section 2 introduces the system model. The optimization framework is subsequently introduced in Sect. 3. The proposed framework will be applied to the analysis of different special cases in Sect. 4 in order to demonstrate the method. The paper concludes in Sect. 5.

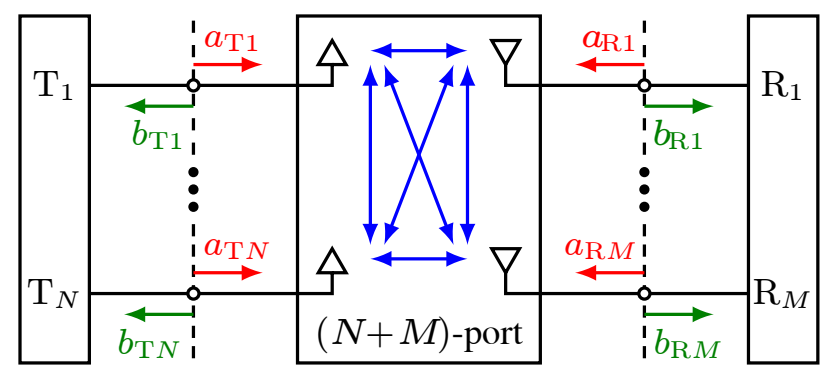

Figure 1. Modeling a MIMO WPT system by means of the network theory.

Notation Column vectors are denoted by bold italic letters and $\|\cdot\|$ is the Euclidean norm of a vector. Matrices are denoted by capitalized bold letters. The matrices $\mathbf{I}_{N}$ and $\mathbf{0}_{N \times M}$ denote the $N \times N$-identity matrix and the $N \times M$-zero matrix, respectively. The superscripts $*{ }^{\mathrm{T}}$ and ${ }^{-1}$ denote the conjugate complex, the transposition and the matrix inverse, respectively.

\section{System model}

A WPT system having $N$ transmit antennas and $M$ receive antennas is considered, see Fig. 1. Here, the MIMO channel is modeled as an $(N+M)$-port, the transmitter is an active $N$-port and the receiver is a passive $M$-port. Since WPT systems reasonably operate at a single transmission frequency, see the discussions in Grover and Sahai (2010), the following investigations are done for one operating frequency as well. The channel input vector $\boldsymbol{a}$, which contains both the incident waves at the transmitter side $\boldsymbol{a}_{\mathrm{Tx}}=\left[a_{\mathrm{T} 1} \cdots a_{\mathrm{TN}}\right]^{\mathrm{T}}$ and at the receiver side $\boldsymbol{a}_{\mathrm{Rx}}=\left[a_{\mathrm{R} 1} \cdots a_{R M}\right]^{\mathrm{T}}$, reads

$\boldsymbol{a}=[\underbrace{a_{\mathrm{T} 1} \cdots a_{\mathrm{T} N}}_{\boldsymbol{a}_{\mathrm{Tx}}^{\mathrm{T}}} \underbrace{a_{\mathrm{R} 1} \cdots a_{\mathrm{R} M}}_{\boldsymbol{a}_{\mathrm{Rx}}^{\mathrm{T}}}]^{\mathrm{T}}$.

Accordingly, the channel output vector is defined as

$\boldsymbol{b}=[\underbrace{b_{\mathrm{T} 1} \cdots b_{\mathrm{T} N}}_{\boldsymbol{b}_{\mathrm{Tx}}^{\mathrm{T}}} \underbrace{b_{\mathrm{R}} \cdots b_{\mathrm{R} M}}_{\boldsymbol{b}_{\mathrm{Rx}}^{\mathrm{T}}}]^{\mathrm{T}}$

and contains the reflected waves at the transmitter side $\boldsymbol{b}_{\mathrm{Tx}}=\left[b_{\mathrm{T} 1} \cdots b_{\mathrm{TNN}}\right]^{\mathrm{T}}$ and at the receiver side $\boldsymbol{b}_{\mathrm{Rx}}=\left[b_{\mathrm{R} 1} \cdots b_{\mathrm{R} M}\right]^{\mathrm{T}}$. Assuming the multiport to be linear, the relation between the incident waves in Eq. (1) and the reflected waves in Eq. (2) can be expressed by scattering parameters (Pozar, 2012) in form of a block matrix $\mathbf{S}$, reading

$\left(\begin{array}{c}\boldsymbol{b}_{\mathrm{Tx}} \\ \boldsymbol{b}_{\mathrm{Rx}}\end{array}\right)=\underbrace{\left(\begin{array}{ll}\mathbf{S}_{\mathrm{TT}} & \mathbf{S}_{\mathrm{TR}} \\ \mathbf{S}_{\mathrm{RT}} & \mathbf{S}_{\mathrm{RR}}\end{array}\right)}_{\mathbf{S}}\left(\begin{array}{c}\boldsymbol{a}_{\mathrm{Tx}} \\ \boldsymbol{a}_{\mathrm{Rx}}\end{array}\right)$,

where the matrices $\mathbf{S}_{\mathrm{TT}} \in \mathcal{C}^{N \times N}$ and $\mathbf{S}_{\mathrm{RR}} \in \mathcal{C}^{M \times M}$ represent the antenna cross-couplings at the transmitter array 
and the receiver array, respectively, and $\mathbf{S}_{\mathrm{RT}} \in \mathcal{C}^{M \times N}$ and $\mathbf{S}_{\mathrm{TR}} \in \mathcal{C}^{N \times M}$ represent the MIMO channel in forward and backward direction, respectively.

The transmitter radiates the power

$$
\begin{aligned}
P_{\mathrm{Tx}} & =\frac{1}{2}\left(\left\|\boldsymbol{a}_{\mathrm{Tx}}\right\|^{2}-\left\|\boldsymbol{b}_{\mathrm{Tx}}\right\|^{2}\right) \\
& =\frac{1}{2} \boldsymbol{a}^{* \mathrm{~T}} \underbrace{\left(\begin{array}{cc}
\mathbf{I}_{N}-\mathbf{S}_{\mathrm{TT}}^{* \mathrm{~T}} \mathbf{S}_{\mathrm{TT}} & -\mathbf{S}_{\mathrm{TT}}^{* \mathrm{~T}} \mathbf{S}_{\mathrm{TR}} \\
-\mathbf{S}_{\mathrm{TR}}^{* \mathrm{~T}} \mathbf{S}_{\mathrm{TT}} & -\mathbf{S}_{\mathrm{TR}}^{* \mathrm{~T}} \mathbf{S}_{\mathrm{TR}}
\end{array}\right)}_{\mathbf{B}} \\
& \geq 0 .
\end{aligned}
$$

It is worth noticing that in Eq. (4) some manipulations are done in order to obtain the transmitted power $P_{\mathrm{Tx}}$ as a function of the channel input vector $\boldsymbol{a}$ alone. Furthermore, the radiated power $P_{\mathrm{Tx}}$ is constrained to be non-negative in order to ensure the that power is transferred from the transmitter into the multiport. Analogously, the received power $P_{\mathrm{Rx}}$, which is the power dissipated in the load at the receiver side, is

$$
\begin{aligned}
P_{\mathrm{Rx}} & =\frac{1}{2}\left(\left\|\boldsymbol{b}_{\mathrm{Rx}}\right\|^{2}-\left\|\boldsymbol{a}_{\mathrm{Rx}}\right\|^{2}\right) \\
& =\frac{1}{2} \boldsymbol{a}^{* \mathrm{~T}} \underbrace{\left(\begin{array}{cc}
\mathbf{S}_{\mathrm{RT}}^{* \mathrm{~T}} \mathbf{S}_{\mathrm{RT}} & \mathbf{S}_{\mathrm{RT}}^{* \mathrm{~T}} \mathbf{S}_{\mathrm{RR}} \\
\mathbf{S}_{\mathrm{RR}}^{* \mathrm{~T}} \mathbf{S}_{\mathrm{RT}} & \mathbf{S}_{\mathrm{RR}}^{* \mathrm{~T}} \mathbf{S}_{\mathrm{RR}}-\mathbf{I}_{M}
\end{array}\right)}_{\mathbf{A}}) \boldsymbol{a} \\
& \geq 0 .
\end{aligned}
$$

For convenience, the Hermitian matrices

$\mathbf{A} \in \mathcal{C}^{(N+M) \times(N+M)}$ and $\mathbf{B} \in \mathcal{C}^{(N+M) \times(N+M)}$ are introduced.

\section{Optimization problem}

The power transfer efficiency (PTE) $\eta$ is defined in accordance to (Hui et al., 2014; Yuan et al., 2013) as the ratio of the dissipated power in the load $P_{\mathrm{Rx}}$ to the total transmitted power $P_{\mathrm{Tx}}$. The total system efficiency is not discussed within the following optimization so that power losses in the source are not considered here, see the discussion in Hui et al. (2014).

Using the Eqs. (1)-(5), the PTE can be written as

$\eta=\frac{\text { dissipated power in the load } P_{\mathrm{Rx}}}{\text { total transmitted power } P_{\mathrm{Tx}}}=\frac{\boldsymbol{a}^{* \mathrm{~T}} \mathbf{A} \boldsymbol{a}}{\boldsymbol{a}^{* \mathrm{~T}} \mathbf{B} \boldsymbol{a}}$,

which is a generalized Rayleigh quotient (Moon and Stirling, 2000). On the basis of Eq. (6), the optimization problem to be solved reads

$\eta_{i}=\max _{\boldsymbol{a}} \eta$

subject to: $P_{\mathrm{Rx}}, P_{\mathrm{Tx}} \geq 0$.

Equating the gradient of the generalized Rayleigh quotient $\eta$ to zero, one finds that $\eta$ reaches an extreme value if $\boldsymbol{a}$ solves the generalized eigenvalue problem

$\mathbf{A} \boldsymbol{a}_{i}=\eta_{i} \mathbf{B} \boldsymbol{a}_{i}$.

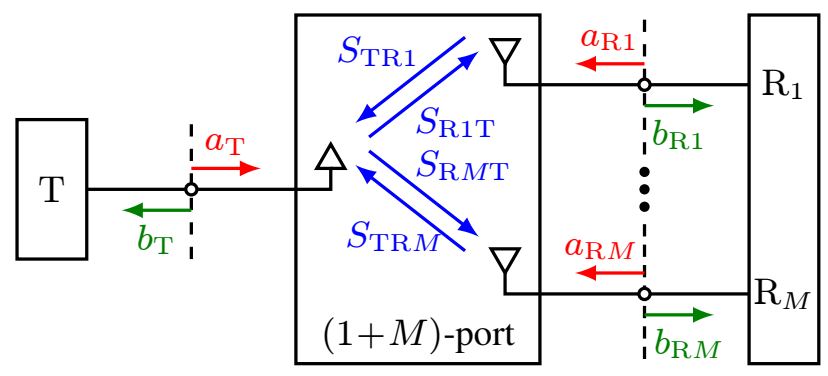

Figure 2. Modeling a SIMO WPT system by means of the network theory. Cross-coupling at the receive antenna array is neglected. $\mathbf{S}_{\mathrm{TR}}=\left[S_{\mathrm{TR} 1} \cdots S_{\mathrm{TR} M}\right]^{\mathrm{T}}=\mathbf{0}_{1 \times M}$ holds true for an unidirectional channel model.

Notice that the multiport is assumed to be passive, i.e. $\boldsymbol{a}^{* \mathrm{~T}} \boldsymbol{a} \geq \boldsymbol{b}^{* \mathrm{~T}} \boldsymbol{b}$, so that together with the constraints $P_{\mathrm{Rx}}, P_{\mathrm{Tx}} \geq 0$, only the solutions of the generalized eigenvalue problem of Eq. (8) with $0 \leq \eta_{i} \leq 1$ are relevant for the solution of Eq. (7). Corresponding solutions are denoted as WPT in the forward direction in the following. If the MIMO channel can be considered as unidirectional, then the constraints become irrelevant.

\section{Examples}

\subsection{SIMO WPT systems}

First, SIMO WPT systems, see Fig. 2, are considered to illustrate the convenience of the new approach. It is assumed at this point that the receive antennas are well in the farfield of the transmit antenna and that power transmission in the forward direction is possible, i.e., $\left\|\mathbf{S}_{\mathrm{RT}}\right\|>0$. This motivates an unidirectional channel model with $\mathbf{S}_{\mathrm{TR}}=\mathbf{0}_{1 \times M}$ (Ivrlac and Nossek, 2010). For simplification, receiver-side cross-couplings are neglected at this point and the ports are assumed to be ideally matched. Then, it is $S_{\mathrm{TT}}=0$, $\mathbf{S}_{\mathrm{RR}}=\mathbf{0}_{M \times M}$. Having said this, the nonsingular Hermitian matrix of Eq. (5) reads

$\mathbf{A}=\left(\begin{array}{cc}\left\|\mathbf{S}_{\mathrm{RT}}\right\|^{2} & \mathbf{0}_{1 \times M} \\ \mathbf{0}_{M \times 1} & -\mathbf{I}_{M}\end{array}\right)$,

and the Hermitian matrix of Eq. (4) reads

$\mathbf{B}=\left(\begin{array}{cc}1 & \mathbf{0}_{1 \times M} \\ \mathbf{0}_{M \times 1} & \mathbf{0}_{M \times M}\end{array}\right)=\left(\begin{array}{c}1 \\ \mathbf{0}_{M \times 1}\end{array}\right)\left(\begin{array}{ll}1 & \mathbf{0}_{1 \times M}\end{array}\right)$.

Since the inverse of the matrix $\mathbf{A}$ exits, the generalized eigenvalue problem of Eq. (8) reduces to the specific eigenvalue problem

$\frac{1}{\eta_{i}} \boldsymbol{a}_{i}=\mathbf{A}^{-1} \mathbf{B} \boldsymbol{a}_{i}$ 
Using the matrix decomposition of Eq. (10), the specific eigenvalue problem of Eq. (11) yields

$$
\frac{1}{\eta_{i}} \boldsymbol{a}_{i}=\frac{1}{\eta_{i}}\left(\begin{array}{l}
a_{\mathrm{T}} \\
\boldsymbol{a}_{\mathrm{Rx}}
\end{array}\right)=\frac{1}{\left\|\mathbf{S}_{\mathrm{RT}}\right\|^{2}}\left(\begin{array}{l}
a_{\mathrm{T}} \\
\mathbf{0}_{M \times 1}
\end{array}\right) .
$$

As a result, only one relevant solution with the eigenvalue

$\eta_{1}=\left\|\mathbf{S}_{\mathrm{RT}}\right\|^{2}$

is obtained. Since $0 \leq \eta_{1} \leq 1$, the maximum PTE is accordingly achieved in the forward direction. Straightforwardly, the optimal incident and reflect Eqs. (12) and (3), reading

$$
\begin{gathered}
\boldsymbol{a}_{1}=[\underbrace{a_{\mathrm{T}}^{a_{\mathrm{T}}^{\mathrm{T}}}}_{\boldsymbol{a}_{\mathrm{Tx}}^{\mathrm{T}}} \underbrace{\mathbf{0}_{1 \times M}^{\mathrm{T}}}]^{\mathrm{T}}, \\
\boldsymbol{b}_{1}=\left[\begin{array}{cc}
\boldsymbol{b}_{\mathrm{Tx}}^{\mathrm{T}} & \underbrace{a_{\mathrm{T}} \mathbf{S}_{\mathrm{RT}}^{\mathrm{T}}}_{\boldsymbol{b}_{\mathrm{Rx}}^{\mathrm{T}}}
\end{array}\right]^{\mathrm{T}} .
\end{gathered}
$$

As can be seen, an optimal receiver multiport for this scenario must realize zero reflection. However, this does not hold true for arbitrary MIMO channels, which will be illustrated below in the example of receiver-side cross-couplings.

Setting $M=1$ in the above discussion directly yields the SISO solution, i.e., $\eta_{1}=\left|S_{\mathrm{RT}}\right|^{2}$ and $\boldsymbol{a}_{1}=\left[\begin{array}{ll}1 & 0\end{array}\right]^{\mathrm{T}}$. This corresponds to the literature when transmitter and receiver matching is considered for the PTE optimization of SISO WPT systems, compare e.g., Yuan et al. (2010); Agbinya (2012).

For simplicity, the number of receive antennas is set to $M=2$ in the following discussion. A special case of crosscouplings between the receive antennas is $S_{23}=S_{32}=K$, where the coupling is assumed to be reciprocal and quantified by $0 \leq|K|<1$. Reflections are still neglected so that

$\mathbf{S}_{\mathrm{RR}}=\left(\begin{array}{cc}0 & K \\ K & 0\end{array}\right)$,

which modifies the matrix A of Eq. (9) to

$\mathbf{A}=\left(\begin{array}{ccc}\left\|\mathbf{S}_{\mathrm{RT}}\right\|^{2} & K S_{21}^{*} & K S_{21}^{*} \\ K^{*} S_{31} & K^{2}-1 & 0 \\ K^{*} S_{21} & 0 & K^{2}-1\end{array}\right)$

Applying again the discussed solution of Eq. (12), one obtains the relevant solution

$\frac{1}{\eta_{i}} \boldsymbol{a}_{i}=\frac{1}{\eta_{i}}\left(\begin{array}{c}a_{\mathrm{T}} \\ a_{\mathrm{R} 1} \\ a_{\mathrm{R} 2}\end{array}\right)=\frac{1-|K|^{2}}{\left\|\mathbf{S}_{\mathrm{RT}}\right\|^{2}}\left(\begin{array}{c}a_{\mathrm{T}} \\ \frac{a_{\mathrm{T}} K^{*} S_{31}}{1-|K|^{2}} \\ \frac{a_{\mathrm{T}} K^{*} S_{21}}{1-|K|^{2}}\end{array}\right)$,

with the corresponding eigenvalue

$\eta_{1, \mathrm{c}}=\frac{\left\|\mathbf{S}_{\mathrm{RT}}\right\|^{2}}{1-|K|^{2}}$ and the optimal incident and reflected waves

$\boldsymbol{a}_{1, \mathrm{c}}=[\underbrace{a_{\mathrm{T}}}_{a_{\mathrm{T} x}} \underbrace{\frac{a_{\mathrm{T}} K^{*} S_{31}}{1-|K|^{2}}}_{a_{\mathrm{R} 1}} \underbrace{\frac{a_{\mathrm{T}} K^{*} S_{21}}{1-|K|^{2}}}_{a_{\mathrm{R} 2}}]^{\mathrm{T}}$,
$\boldsymbol{b}_{1, \mathrm{c}}=[\underbrace{0}_{b_{\mathrm{Tx}}} \underbrace{\frac{a_{\mathrm{T}} S_{21}}{1-|K|^{2}}}_{b_{\mathrm{R} 1}} \underbrace{\frac{a_{\mathrm{T}} S_{31}}{1-|K|^{2}}}_{b_{\mathrm{R} 2}}]^{\mathrm{T}}$.

As can be seen by comparing to Eq. (13), the cross-couplings enhance the PTE in this particular case. Note that the incident waves $\boldsymbol{a}_{\mathrm{Rx}}$ at the receiver side are now nonzero due to the antenna cross-couplings. This may become crucial if $\left|a_{\mathrm{R} 1}\right|>\left|b_{\mathrm{R} 1}\right|$ or $\left|a_{\mathrm{R} 2}\right|>\left|b_{\mathrm{R} 2}\right|$, respectively, since then one receive antenna has to be switched to transmit mode in order to maximize the PTE. The investigation of this phenomenon is beyond the scope of this paper. Thus, the influence of antenna cross-couplings on the PTE may be studied in more detail in a future work.

\subsection{MISO WPT systems}

Next, a bidirectional channel with $\left\|\mathbf{S}_{\mathrm{RT}}\right\|>0$ and $\left\|\mathbf{S}_{\mathrm{TR}}\right\|>0$ is considered for the scenario of Sect. 4.1, see Fig. 2. Whereas Eq. (9) still holds, Eq. (10) changes to

$\mathbf{B}=\left(\begin{array}{cc}1 & \mathbf{0}_{1 \times M} \\ \mathbf{0}_{M \times 1} & -\mathbf{S}_{\mathrm{TR}}^{* \mathrm{~T}} \mathbf{S}_{\mathrm{TR}}\end{array}\right)$

so that the matrix $\mathbf{B}$ cannot be decomposed anymore. As a consequence, the characteristic polynomial

$$
\left(\mathbf{I}_{N+M}-\eta_{i} \mathbf{A}^{-1} \mathbf{B}\right) \boldsymbol{a}=0
$$

of the specific eigenvalue problem of Eq. (11) has to be evaluated. Equating the determinant to zero followed by a substitution of the Eqs. (9) and (22) yields

$\eta_{i}^{N+M-2}\left(\left\|\mathbf{S}_{\mathrm{RT}}\right\|^{2}-\eta_{i}\right)\left(\left\|\mathbf{S}_{\mathrm{TR}}\right\|^{2} \eta_{i}-1\right)=0$

As a result, one obtains $\eta_{0}=0$, which is a semisimple eigenvalue having a multiplicity equal to $N+M-2$, and two nonzero eigenvalues. The first nonzero eigenvalue $\eta_{1}=\left\|\mathbf{S}_{\mathrm{RT}}\right\|^{2}$ agrees with the solution in Eq. (13) and corresponds to the WPT in the forward direction, which is due to the assumed matched ports and the absence of antenna array cross-couplings. Again, this holds true only for the considered scenario.

The second nonzero eigenvalue $\eta_{2}=1 /\left\|\mathbf{S}_{\mathrm{TR}}\right\|^{2}>1$ is no relevant solution for the WPT in the forward direction and one easily proofs that $P_{\mathrm{Rx}}, P_{\mathrm{Tx}} \leq 0$, which violates the constraints of Eq. (7). However, it is worth noting that this solution maximizes the PTE in the backward direction, i.e., it solves the maximization problem 


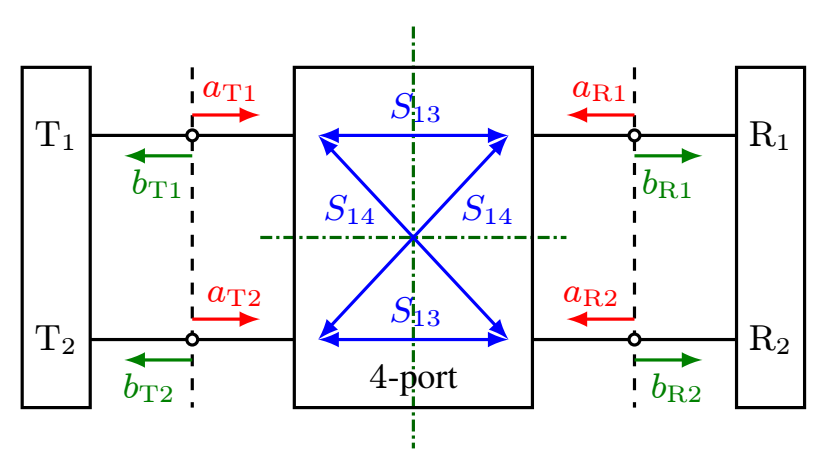

Figure 3. Modeling the WPT over a $(2 \times 2)$ channel having a double-symmetric scattering matrix.

$\max _{a} \frac{1}{\eta}$

subject to : $P_{\mathrm{Rx}}, P_{\mathrm{Tx}} \leq 0$.

This is of interest if the transmitter and receiver functionality shall be exchanged. Then, $1 / \eta_{2}=\left\|\mathbf{S}_{\mathrm{TR}}\right\|^{2}$ is the maximum PTE, which can be achieved in backward direction over the corresponding $(M \times 1)$-MISO channel. Substituting the Eqs. (9) and (22) as well as $\eta_{2}$ into the generalized eigenvalue problem of Eq. (8), one easily proofs that

$$
\mathbf{a}_{2}=[\underbrace{0}_{\boldsymbol{a}_{\mathrm{Tx}}^{\mathrm{T}}} \underbrace{\mathbf{S}_{\mathrm{TR}}^{*}}_{\boldsymbol{a}_{\mathrm{Rx}}^{\mathrm{T}}}]^{\mathrm{T}}
$$

and

$$
\mathbf{b}_{2}=[\underbrace{\left\|\mathbf{S}_{\mathrm{TR}}\right\|^{2}}_{\boldsymbol{b}_{\mathrm{Tx}}^{\mathrm{T}}} \underbrace{\mathbf{0}_{1 \times M}}_{\boldsymbol{b}_{\mathrm{Rx}}^{\mathrm{T}}}]^{\mathrm{T}}
$$

describe the corresponding operating point for the WPT system. This solution is known as "energy beamforming" in the literature, see Zhang and Ho (2013).

\subsection{Directional coupler}

Furthermore, a $(2 \times 2)$-MIMO channel is considered in order to discuss the optimization framework. For simplicity, the scattering matrix is considered to be double-symmetric, i.e., $S_{m n}=S_{n m}, \forall m, n=1 \ldots N$, see Fig. 3. Again, the ports are matched and both transmitter-side and receiver-side crosscouplings are neglected. The corresponding 4-port scattering matrix is

$\mathbf{S}=\left(\begin{array}{cccc}0 & 0 & S_{13} & S_{14} \\ 0 & 0 & S_{14} & S_{13} \\ S_{13} & S_{14} & 0 & 0 \\ S_{14} & S_{13} & 0 & 0\end{array}\right)$.
With the substitution

$\kappa=S_{13} S_{14}^{*}+S_{13}^{*} S_{14}=2 \operatorname{Re}\left(S_{13} S_{14}^{*}\right)$,

the matrix

$$
\mathbf{A}=\left(\begin{array}{cccc}
\left|S_{13}\right|^{2}+\left|S_{14}\right|^{2} & \kappa & 0 & 0 \\
\kappa & \left|S_{13}\right|^{2}+\left|S_{14}\right|^{2} & 0 & 0 \\
0 & 0 & 1 & 0 \\
0 & 0 & 0 & 1
\end{array}\right)
$$

is obtained from Eq. (5) and, analogously, the matrix

$$
\mathbf{B}=\left(\begin{array}{cccc}
1 & 0 & 0 & 0 \\
0 & 1 & 0 & 0 \\
0 & 0 & -\left|S_{13}\right|^{2}-\left|S_{14}\right|^{2} & -\kappa \\
0 & 0 & -\kappa & -\left|S_{13}\right|^{2}-\left|S_{14}\right|^{2}
\end{array}\right)
$$

is obtained from Eq. (4). Again, the inverse of the matrix $\mathbf{B}$ exits so that the corresponding specific eigenvalue problem has to be solved. Regarding the WPT in the forward direction, the eigenvectors are

$\eta_{1,2}=\left|S_{13}\right|^{2}+\left|S_{14}\right|^{2} \pm \kappa$

with the corresponding channel vectors

$$
\begin{aligned}
& \boldsymbol{a}_{1,2}=[\underbrace{ \pm 1}_{\boldsymbol{a}_{\mathrm{Tx}}^{\mathrm{T}}} \underbrace{ \pm}_{\boldsymbol{a}_{\mathrm{Rx}}^{\mathrm{T}}} \underbrace{0},
\end{aligned}
$$

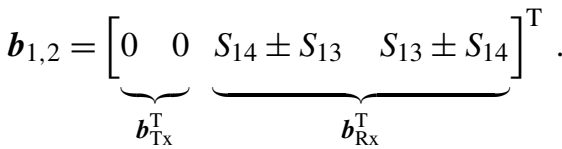

As can be seen, two relevant solutions for the optimal incident waves can be found. The basic difference is in the argument of $\boldsymbol{a}_{\mathrm{T} 1}$. If

$\left|\arg \left(S_{13}\right)+\arg \left(S_{14}\right)\right|<\frac{\pi}{2}$,

i.e., the angle between $S_{13}$ and $S_{14}$ is smaller than $\pi / 2$, $\kappa$ in Eq. (32) is positive and the optimal way for WPT in the forward direction is to transmit the same signal via both transmit antennas. If contrarily the angle is larger than $\pi / 2$, $\kappa$ in Eq. (32) is negative and the optimal way is to transmit two $\pi$-phase shifted signals, each via one transmit antenna.

Due to the symmetry, the eigenvalues for the backward direction can be easily found by using

$\eta_{3,4}=\frac{1}{\eta_{1,2}}$.

Accordingly, the corresponding optimal incident and reflected waves are obtained by exchanging $\boldsymbol{a}_{\mathrm{Tx}}$ and $\boldsymbol{a}_{\mathrm{Rx}}$ in Eq. (33) and, respectively, $\boldsymbol{b}_{\mathrm{Tx}}$ and $\boldsymbol{b}_{\mathrm{Rx}}$ in Eq. (34). The discussion of the solution is analogous to the discussion of the WPT in the forward direction. 
Now, the $(2 \times 2)$-MIMO system described by the scattering matrix of Eq. (28) is further assumed to be lossless, i.e., $\mathbf{S S}^{* \mathrm{~T}}=\mathbf{I}_{4}$. Then, the matrix of Eq. (28) equals the scattering matrix of an ideal directional coupler (Maloratsky, 2003). The relative phase difference of the ideal coupler's output is $\pm \pi / 2$ so that the constraint

$\arg \left(S_{13}\right)+\arg \left(S_{14}\right)= \pm \frac{\pi}{2}$

follows. Without loss of generality, $\arg \left(S_{13}\right)=0$ is assumed in the following. Then and by means of Eq. (37), the equality

$S_{14}=-S_{14}^{*}$

holds so that

$\kappa=0$,

compare Eq. (29). Using the Eqs. (37)-(39), the matrices

$\mathbf{A}=\mathbf{B}=\left(\begin{array}{cc}\mathbf{I}_{2} & \mathbf{0}_{2 \times 2} \\ \mathbf{0}_{2 \times 2} & -\mathbf{I}_{2}\end{array}\right)$

are obtained from the Eqs. (30)-(31), respectively. Since the inverse of the matrix $\mathbf{B}$ still exists, the specific eigenvalue problem

$\mathbf{I}_{4} \mathbf{a}_{i}=\eta_{i} \mathbf{a}_{i}$

is obtained from Eq. (8). As a result, all eigenvalues are equal to one, which is trivial due to the absence of losses in this scenario. Eq. (41) is fulfilled by arbitrary $\boldsymbol{a}_{i}$ so that all possible waves are optimal for the ideal directional coupler.

\section{Conclusions}

This paper discusses an optimization framework for the PTE over general MIMO channels using network theory. This framework yields the maximum achievable PTE and the corresponding operating point.

The proposed framework can be used in order to gain basic insights into the wireless power transfer systems from a theoretical point of view. This is demonstrated in the paper by means of special cases, e.g., SIMO, MISO and a special $(2 \times 2)$-MIMO system. As it is shown, a significant reduction in the analysis effort is obtained, if the considered multiport features certain properties. It is beyond the scope of this paper to entirely discuss all properties so that further investigations may have to be done.

In future work, simulations and real-world measurements may be carried out in order to assess the performance gap of suboptimal system realizations and the herein determined upper bounds. Furthermore, the relevance of the proposed framework for the performance maximization of the combined communication and power transfer over MIMO channels may be investigated.
Data availability. No data sets were used in this article.

Competing interests. The authors declare that they have no conflict of interest.

Edited by: J. Anders

Reviewed by: two anonymous referees

\section{References}

Agbinya, J. I. (Ed.): Wireless Power Transfer, River Publishers, Aalborg, 2012.

Grover, P. and Sahai, A.: Shannon meets Tesla: Wireless information and power transfer, IEEE International Symposium on Information Theory, 2363-2367, 2010.

Hui, S., Zhong, W., and Lee, C.: A Critical Review of Recent Progress in Mid-Range Wireless Power Transfer, IEEE T. Power Electr., 29, 4500-4511, 2014.

Ivrlac, M. and Nossek, J.: Toward a Circuit Theory of Communication, IEEE T. Circuits-I, 57, 1663-1683, 2010.

Kim, J. W., Son, H. C., Kim, D. H., and Kim, K. H.: Analysis of wireless energy transfer to multiple devices using CMT, AsiaPacific Microwave Conference, 2149-2152, 2010.

Lang, H.-D., Ludwig, A., and Sarris, C.: Convex Optimization of Wireless Power Transfer Systems With Multiple Transmitters, IEEE T. Antenn. Propag., 62, 4623-4636, 2014.

Lee, J. and Nam, S.: Fundamental Aspects of Near-Field Coupling Small Antennas for Wireless Power Transfer, IEEE T. Antenn. and Propag., 58, 3442-3449, 2010.

Maloratsky, L.: Passive RF \& Microwave Integrated Circuits, Elsevier Science, Burlington, 2003.

Moon, T. K. and Stirling, W. C.: Mathematical Methods and Algorithms for Signal Processing, Prentice Hall, Upper Saddle River, 2000.

Nguyen, M. Q., Plesa, D., Rao, S., and Chiao, J. C.: A multi-input and multi-output wireless energy transfer system, IEEE MTT-S International Microwave Symposium, 1-3, 2014.

Pozar, D. M.: Microwave Engineering, John Wiley \& Sons, Inc, Hoboken, 4. Edn., 2012.

Wallace, J. W. and Jensen, M. A.: Mutual coupling in MIMO wireless systems: a rigorous network theory analysis, Transactions on Wireless Communications, 3, 1317-1325, 2014.

Wu, M., Chen, Q., and Yuan, Q.: Analysis of near-field power transfer of multi-antenna using multiport scattering parameters, Proceedings of the International Symposium on Antennas \& Propagation, 313-316, 2013.

Xiang, Z. and Tao, M.: Robust Beamforming for Wireless Information and Power Transmission, IEEE Wireless Communications Letters, 1, 372-375, 2012.

Xu, J. and Zhang, R.: A General Design Framework for MIMO Wireless Energy Transfer With Limited Feedback, IEEE T. Signal Proces., 64, 2475-2488, 2016.

Yoon, I.-J. and Ling, H.: Investigation of Near-Field Wireless Power Transfer Under Multiple Transmitters, IEEE Antenn. Wirel. Pr., 10, 662-665, 2011.

Yuan, Q., Chen, Q., Li, L., and Sawaya, K.: Numerical Analysis on Transmission Efficiency of Evanescent Resonant Coupling Wire- 
less Power Transfer System, IEEE T. Antenn. Propag., 58, 17511758, 2010.

Yuan, Q., Wu, M., Chen, Q., and Sawaya, K.: Analysis of nearfield power transfer using scattering parameters, European Conference on Antennas and Propagation, 3073-3075, 2013.

Zargham, M. and Gulak, P.: Maximum Achievable Efficiency in Near-Field Coupled Power-Transfer Systems, IEEE Transactions on Biomedical Circuits and Systems, 6, 228-245, 2012.
Zhang, R. and Ho, C. K.: MIMO Broadcasting for Simultaneous Wireless Information and Power Transfer, IEEE T. Wireless Commun., 12, 1989-2001, 2013. 